\title{
Key Factor of Graphene Localization on Electrical Conductive Properties of Graphene Filled Polyethylene/Polypropylene Composites during Melt Blending
}

\author{
$\mathrm{Ce} \mathrm{Tu}^{1,2}$, Kenji Nagata ${ }^{2,3} \&$ Shouke Yan ${ }^{1}$ \\ ${ }^{1}$ State Key Laboratory of Chemical Resource Engineering, Beijing University of Chemical Technology, China \\ ${ }^{2}$ Department of Materials Science and Engineering, Graduate School of Engineering, Nagoya Institute of \\ Technology, Japan \\ ${ }^{3}$ Institute of Ceramics Research and Education, Nagoya Institute of Technology, Japan \\ Correspondence: Kenji Nagata, Department of Materials Science and Engineering, Graduate School of \\ Engineering, Nagoya Institute of Technology, Japan. E-mail: nagata.kenji@nitech.ac.jp
}

Received: May 4, 2017

Accepted: May 17, 2017

Online Published: June 5, 2017

doi:10.5539/jmsr.v6n3p1

URL: https://doi.org/10.5539/jmsr.v6n3p1

\begin{abstract}
The effect of surfactant-exfoliated graphene (SEG) on the morphology of SEG-filled polyethylene (PE)/ polypropylene (PP) blends has been investigated by image analysis of transmission electron microscope. The electrical conductivity of SEG-filled PE/PP composites strongly depends on the localization of SEG. From theoretical considerations of a previous paper by one of the authors, it is found that the transfer dynamics as well as the stability of different solid nano-fillers at the blends interface reveals a strong dependence on the nano-filler's aspect ratio. The appropriate control of processing conditions and the selective localization of SEG in the PE/PP composites are key tools to design conducting polymer composites. When the selective localization of SEG is optimized at the PE/PP blends interface, the electrical conductivity reaches $1.86 \times 10^{-5} \mathrm{~S} / \mathrm{m}$ for a low percolation at $1 \mathrm{wt} \%$, in sharp compared to $7 \mathrm{wt} \%$ if the localization of SEG has not been optimized.
\end{abstract}

Keywords: polymer-matrix composites (PMCs), electrical properties, rheology, transmission electron microscopy, extrusion

\section{Introduction}

Conducting polymer composite materials (CPCM) (Ponomarenko, Shevchenko, \& Enikolopyan, 1990), which consist of random distribution of conductive fillers throughout the insulating polymer matrix, deserve interest in several application fields. They are used as low-temperature heaters, antistatic materials, and they are very promising for electromagnetic interference shielding materials (Yavari, Rafiee, Rafiee, Yu, \& Koratkar, 2010; Kim, Miura, \& Macosko, 2010).

The choice of the filler and polymer is a key factor in the preparation of CPCM. There must be indeed a good balance between the filler-polymer and the filler-filler interactions. If the filler self-interactions govern, then the fillers prefer to stick together and to form aggregations rather than filler entangle polymer chains. In contrast, an ideal interaction of the polymer to the filler results in an insulating layer around the fillers and prevents the formation of filler aggregates. In this respect, the lasting use of graphene in CPCM makes it become one of the most attractive conducting filler due to its unique two-dimensional structure, large surface area and high aspect ratio, ease of large scale production (top-down methods by exfoliation of graphite), and amazing properties (Geim, 2009; Rao, Sood, Subrahmanyam, \& Govindaraj, 2009). Another major problem in the preparation of CPCM is the fillers content, which have to be as low as possible, otherwise the mixture processing becomes difficult due to the high viscosity, the mechanical properties of the composites are degradation, and the final cost will be high (Kim, Abdala, \& Macosko, 2010).

As proposed by previous work, a non-oxidative method for producing graphene have been reported, surfactant exfoliated graphene (SEG) was prepared by the sonication with the addition of the surfactant. The SEG overcome the limitation of oxide-derived graphene rest on the generation of defective graphene basal planes (vacancy defects) from the exceedingly harsh oxidation process (Shannon, 2012; Pham et al., 2016). However, the SEG was easy to 
formed aggregations in the high-density polyethylene (PE)/polypropylene (PP) melt blends, the poor SEG dispersion resulted in a high electrical percolation threshold of $7 \mathrm{wt} \%$ reported in our previous study (Tu, Nagata, \& Yan, 2015)

It should be pointed out that, among the various combinations of filler localizations and phase structures that can be formed in immiscible binary polymer blend systems, one special structure is especially promising where the fillers are selectively localized at the interface of the co-continuous blends. Such morphology can potentially leads to an ultralow electrical percolation threshold (Potschke, Bhattacharyya, \& Janke, 2003). Hence the localization of the graphene at the blends interface is highly expected to be the ideal pattern to reach the lowest possible electrical percolation thresholds. Meanwhile, Potschke et al. (Goldel, Marmur, Kasaliwal, Potschke, \& Heinrich, 2011) reported the transfer dynamics as well as the stability of different solid fillers at the melting blends interface reveal a strong dependence on the particle's aspect ratio, indicating the low aspect ratio nano-fillers with a significantly higher stability at the blends interface in a recent work entitled as Slim-Fast Mechanism (SFM). In details, the low aspect ratio sphere-like carbon black (CB) shows a higher stability at the poly(styrene acrylonitrile) (SAN)/ polycarbonate (PC) blends interface, compared to the high aspect ratio rod-like carbon nanotubes (CNTs). Wang et al. investigated the effect of aspect ratio on the interfacial stability of CNTs- and graphene-filled PLA/EVA composites, respectively, and observed a better interfacial stability of graphene at the immiscible blends interface compared with CNTs, indicated that the percolation threshold was $0.18 \mathrm{wt} \%$ as the graphene located at the interface, whereas the percolation threshold was $0.68 \mathrm{wt} \%$ for the localization of CNTs at the interface (Shen et al., 2015). Based on these results, the SEG could more easier to be localized at the immiscible blends interface due to its much lower aspect ratio aggregations, the disadvantage will be converted to the advantage.

Hence, this paper mainly aims at investigating the possible strategies for achieving an ideal situation of low electrical percolation thresholds in the case of a composition consisting of SEG, PE and PP, followed by transmission electron microscopy analysis. Electrical conductivity has also been measured in order to draw a relationship between their electrical properties and the morphology of the polymer composites. Our results provide a simple and effective route to remarkably decrease the loading of graphene in the PE/PP composites to achieve the electrical percolation using the concept of SFM. Any valuable appearing strategies might deserve attractiveness in several fields of applications, such as antistatic materials (Yavari et al., 2010), electromagnetic interference shielding (Abolins, Caraher., Bopp, \& Lovgren, 1983), electrodes (Novoselov et al., 2012), and polymer conductive films.

\section{Experimental}

\subsection{Materials}

Pristine expanded graphite flakes EC300 were provided by Ito Graphite Co., Ltd., Japan with an average size of $50 \mu \mathrm{m}$. The surfactants used in this work was the nonionic pluronic surfactant F108 (HO- $\left.\left(\mathrm{C}_{2} \mathrm{H}_{4} \mathrm{O}\right)_{141}\left(\mathrm{C}_{3} \mathrm{H}_{6} \mathrm{O}\right)_{44}\left(\mathrm{C}_{2} \mathrm{H}_{4} \mathrm{O}\right)_{141} \mathrm{H}\right)$ with a molecular weight of $\sim 14.6 \mathrm{kDa}$ (Aldrich). The Polypropylene (NOVATEC-PP, MA3, PP) was supplied by Japan Polypropylene Co., Japan with a density of $0.90 \mathrm{~g} / \mathrm{cm}^{3}$. The high-density Polyethylene (HI-Zex, 2100J, PE) with a density of $0.95 \mathrm{~g} / \mathrm{cm}^{3}$ was purchased from Mitsui Chemicals Inc.(Japan).

\subsection{Preparation of Surfactant Exfoliation Graphene}

SEG was prepared by the Shannon's method from pristine graphite flakes as reported in a previous work (Shannon, 2012). A high concentrated surfactant solution ( $15 \% \mathrm{w} / \mathrm{w} \mathrm{F} 108$ of $400 \mathrm{~mL}$ aqueous solution) was added dropwise to the $15 \% \mathrm{w} / \mathrm{w}$ graphite flakes suspension through mechanical feeding by MASTERflex L/S (Cole-Parmer Instrument Company) at a rate of $1 \mathrm{~mL} / \mathrm{min}$ under sonication at a power of $60 \mathrm{~W}$ using Advanced sonifier 250 (BRANSON Co., Ltd., Japan). Graphene suspensions were subsequently dialyzed against Milli-Q water for at least $48 \mathrm{~h}$ using a $3500 \mathrm{MWCO}$ cellulose ester membrane (Bio Design Inc., U.S.A.). SEG sheets finally were obtained by centrifugation of the suspensions by Centrifuge 5220 (Kubota Co., Ltd., Tokyo, Japan) and then freeze-drying the sediment for $72 \mathrm{~h}$ using FD-5N (Tokyo Rikakikai Co., Ltd., Tokyo, Japan).

\subsection{Preparation of SEG filled PE/PP Composites}

The SEG, PE and PP were first dried at $50^{\circ} \mathrm{C}$ under vacuum for $24 \mathrm{~h}$. The SEG filled PE/PP composites were melt-mixed by a HAAKE MiniLab conical twin-screw extruder with $100 \mathrm{rpm}$ rotational speed at $190^{\circ} \mathrm{C}$. The $\mathrm{PE} / \mathrm{PP}$ polymer blend composition weight ratio was $60 / 40$ based on previous work (Nagata, Kimura, Takahashi, \& Kinoshita, 2002). The extrudates were compression-molded at $190^{\circ} \mathrm{C}$ under 2.2MPa to obtain samples for testing.

Our previous study verified that the SEG are selectively dispersed in the PE phase rather than in PP phase. One possibility to explain that selective localization behavior is given by the Young's theory (Young, 1805): fillers are 
expected to be selectively located in one of the two polymer phases where the polymer has a lower interfacial tension with the filler. Taking that into account, the selective localization of SEG in the PE phase indicates a lower interfacial tension between PE and SEG. Commonly, the dominating influence of interfacial tension effects and thermodynamic driving forces on the localization behavior is generally accepted specifically for nano-scale fillers (Goldel et al., 2011). Therefore, if the SEG was first mixed with the less favorable PP, the SEG will be transferred from the PP phase to the PE phase due to the thermodynamic driving force during the second step for mixing (SEG/PP) with PE, while some of them would reach their equilibrium position to locate at the PE/PP blends interface.

Two mixing sequences were thus adopted, and the interrelation between the SEG localization behavior and electrical properties of the composites could be investigated:

(i) (SEG/PE/PP) composite means that SEG, PE and PP were melt-mixd simultaneously for $15 \mathrm{~min}$.

(ii) (SEG/PP)/PE composite means that PP was firstly mixed with SEG for 5 min and then the premixed SEG/PP masterbatch was mixed with PE for $10 \mathrm{~min}$.

\subsection{Characterization}

A JEM-z2500 Transmission electron microscope (TEM, JEOL) at an accelerating voltage of $200 \mathrm{kV}$ was used to observe the microstructures of SEG and SEG filled PE/PP composites. SEG sheets were dispersed in $\mathrm{N}, \mathrm{N}$-dimethylformamide by ultra-sonication and the solution was dropped on a elastic carbon-support film grid. SEG filled PE/PP composites were cryogenically cut with a diamond knife at $-140^{\circ} \mathrm{C}$ for ultrathin sections thinner than $80 \mathrm{~nm}$ by LEICA EM FC7 (Leica Instruments, Inc., GER). A Multimode8, Nanoscope V (BRUKER Instruments, Inc., GER) Atomic force microscope (AFM) in tapping mode was conducted with a special emphasis on the lateral dimension and thickness of the SEG. AFM samples were prepared by spin-coating the $\mathrm{SEG} / N, N$-dimethyl-formamide suspensions onto silicon substrates. Electrical volume conductivity test was performed using an Ultra High Resistance Meter (Advantest R8340A) at an input voltage of 10V. The sample was prepared in a dimension of $\phi 50 \times 0.5 \mathrm{~mm}$. To reduce contact resistance with the measuring electrodes, silver conductive paste was painted on the sample surface. All of the conductivity measurements were carried out at ambient temperature. The Rheological properties of the composite samples were performed at $190{ }^{\circ} \mathrm{C}$ on a VAR-50 Rheometer (JASCO Co., Tokyo, Japan) at using a parallel plate geometry with $25 \mathrm{~mm}$ diameter plates in a nitrogen atmosphere. The measurements were performed in dynamic mode with setting the gap at $0.85 \mathrm{~mm}$. The frequency sweep was performed from 0.01 to $90 \mathrm{rad} / \mathrm{s}$.

\section{Results and Discussion}

\subsection{Graphene Sheets}

Figure 1a shows a TEM image of the produced SEG sheets using the method described above. A significant distribution of lateral sheet size can be observed, and the SEG sheets are predominantly few-layers graphene. To complement the TEM image, AFM was also performed to characterize the structure of the SEG sheets. AFM analysis (Figure 1b) shows that the SEG sheets with an average thickness of about $2.0 \mathrm{~nm}$, which are composed of ca. 5-10 individual graphene layers (considering that the thickness of one layer graphene is equal to $0.34 \mathrm{~nm}$ (Stankovich et al., 2010)). The size of SEG was mostly smaller than $0.1 \mu \mathrm{m}^{2}$.

\subsection{Electrical Conductivity}

The influence of compounding sequences on the electrical conductivity of SEG filled PE/PP (60/40) composites was illustrated by Figure 2, it is clear that (SEG/PP)/PE composites are more effective in improving the electrical conductivity of PE/PP blends than (SEG/PE/PP) composites, evidenced by lower percolation threshold and higher electrical conductivity. The (SEG/PP)/PE composites show a lower percolation threshold of $4 \mathrm{wt} \%$, the conductivity is $2.2 \times 10^{-5} \mathrm{~S} / \mathrm{m}$, which is higher than the antistatic criterion of $10^{-6} \mathrm{~S} / \mathrm{m}$. On the contrary, (SEG/PE/PP) composites show a higher percolation threshold of $7 \mathrm{wt} \%$. These results indicate the (SEG/PP)/PE composites formed a more effective conductive network for the transport of electrons throughout the matrix. 

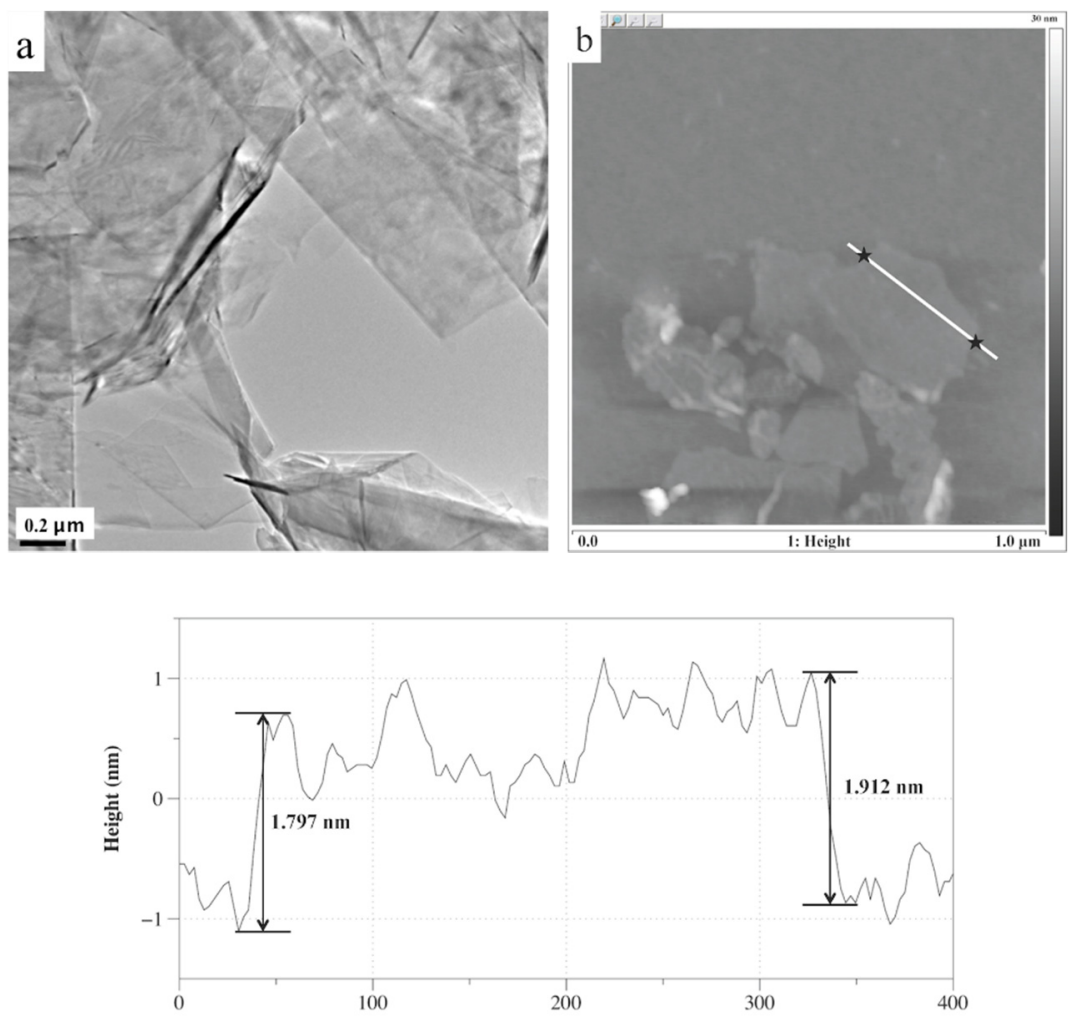

Figure 1. (a) TEM image of SEG sheets, (b) AFM analysis of SEG sheets on Si substrate

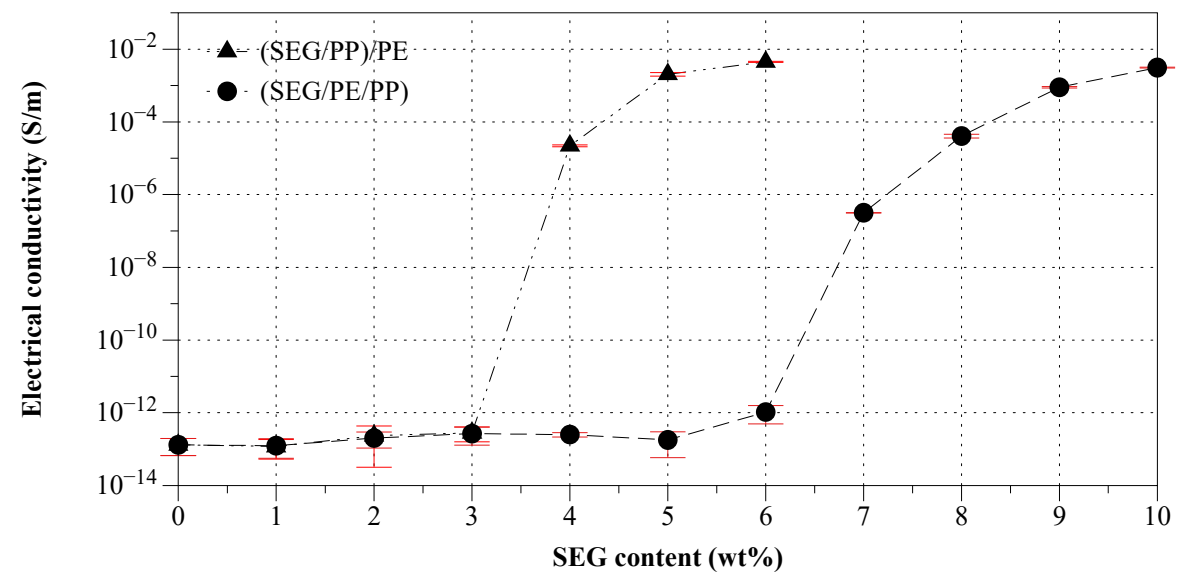

Figure 2. Electrical conductivity versus SEG contents in the PE/PP (60/40) composites doped with SEG (These composites have been prepared with two different sequences: (SEG/PE/PP), (SEG/PP)/PE.)

\subsection{Composites Morphology}

To investigate the percolation behavior of graphene-filled composites, graphene dispersion and localization must be taken into consideration. TEM was used to evaluate the dispersion quality and localization of SEG in the PE/PP composites prepared two different sequences. Typical two-phase structure has been found from TEM micrographs (Figure 3), in which the light and dark parts correspond to PP and PE phases. As shown in Figure 3a, for the (SEG/PE/PP) composites, areas highly concentrated with SEG stacks in the PE phase can be clearly observed, which are distinguished from less SEG dense packed areas, suggesting local concentration fluctuation. The aggregation may be induced by the smaller interaction between non-polar PE matrix and graphene surface, which limits the thermodynamic diffusion of polymer chains into the intragalleries of graphene and makes it difficult to 
break up the aggregates (Kim et al., 2011). As for the (SEG/PP)/PE composites (Figure 3b), SEG were randomly dispersed in PE and PP phase with small aggregates, and most SEG tend to dispersed in PE phase and only a few in PP phase. Whereas it is noteworthy that the some of SEG clearly selectively located at the interface of PE/PP blends, which the morphology is expected to be the ideal scenario to reach the lowest possible electrical percolation thresholds (Gubbels et al., 1998). SEG sheets transfer through the PE/PP blends interface occurs when the SEG are initially located in the less favorable blends phase (PP). When the flow field induces contact of interface and SEG, the molecules of the favorable blends phase (PE) that are close to the interface will advance on the SEG sheets surface and thus, SEG were transferred from PP to PE phase due to the thermodynamic driving force, while some of the SEG approached their equilibrium position at the PE/PP blends interface. The graphene located at a polymer blends interface strongly influence conductivity and can substantially decrease the percolation threshold (Thongruang, Spontak, \& Balik, 2002). That results explain why the electrical properties of the (SEG/PP)/PE composites are markedly superior to the (SEG/PE/PP) composites.
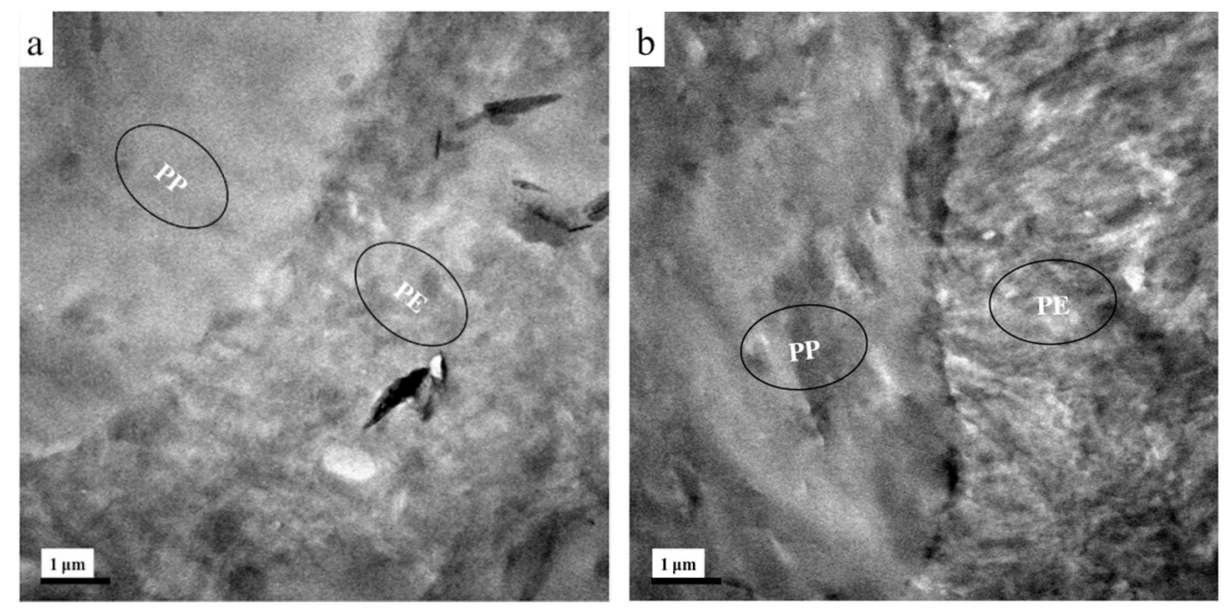

Figure 3. TEM micrographs of 4 wt \% SEG filled (a) (SEG/PE/PP) composite, (b) (SEG/PP)/PE composite (The $\mathrm{PP}$ and $\mathrm{PE}$ phases are indicated by the ellipses.)

\subsection{Fine-Tuning of (SEG/PP)/PE Composites}

According to Potschke's theoretical concept of the SFM (Goldel et al., 2011), for nano-fillers with low aspect ratio, a significantly higher stability at the blends interface was concluded. Meanwhile, the real nano-fillers aspect ratio is highly relevant for melt blending processes (Alig et al., 2012). Thus, more apparent SEG aggregations would lead to lower aspect ratio, higher interfacial stability, more SEG would be located at the blends interface, resulting in a better electrical conductive network. It was well known that, decreasing rotational speed results in lower shear stress levels and lower fragmentation numbers, so that the fast propagating dispersion mechanisms are restrained (Socher et al., 2011). To obtain more aggregations of SEG, the rotational speed of SEG and PP in the first mixing step was decreased from $100 \mathrm{rpm}$ to $50 \mathrm{rpm}$.

The TEM micrographs of 3 wt \% SEG were introduced into PE/PP (60/40) blends using (SEG/PP)/PE sequence run at different mixing rotational speed of SEG and PP in $100 \mathrm{rpm}, 50 \mathrm{rpm}$ were shown in Figure 4. The lower rotational speed (SEG/PP)/PE composite (Figure 4b) exhibits more SEG aggregations formed in PE/PP blends, and more SEG aggregations clearly selective located at the PE/PP blends interface as compared to the higher rotational speed (SEG/PP)/PE composite (Figure 4a). The above results conform to previous explanations of lower aspect ratio nano-fillers or larger aggregations exhibit higher interfacial stability at blends interface, and proved the accuracy of assumption, got a preferable localization of SEG at the PE/PP blends interface. Figure 4c shows the effect of mixing rotational speed of SEG and PP on electrical conductivities of $3 \mathrm{wt} \%$ SEG filled (SEG/PP)/PE composites. The higher rotational speed (SEG/PP)/PE composite shows an insulation properties due to its imperfect conductive network structure. On the contrary, the lower rotational speed (SEG/PP)/PE composite reasonably exhibits a higher electrical conductivity, which could be attributed to a better localization of SEG at the PE/PP blends interface, resulted in a well-developed conductive network formed with the same loading of SEG. However, it should be pointed out that still a small portion of SEG aggregations remained in the PP phase at the lower rotational speed (SEG/PP)/PE composite (Figure $4 \mathrm{~b}$ ), indicated that a more effective localization of SEG at the $\mathrm{PE} / \mathrm{PP}$ blends interface is highly expected to reach a lower possible percolation threshold. 

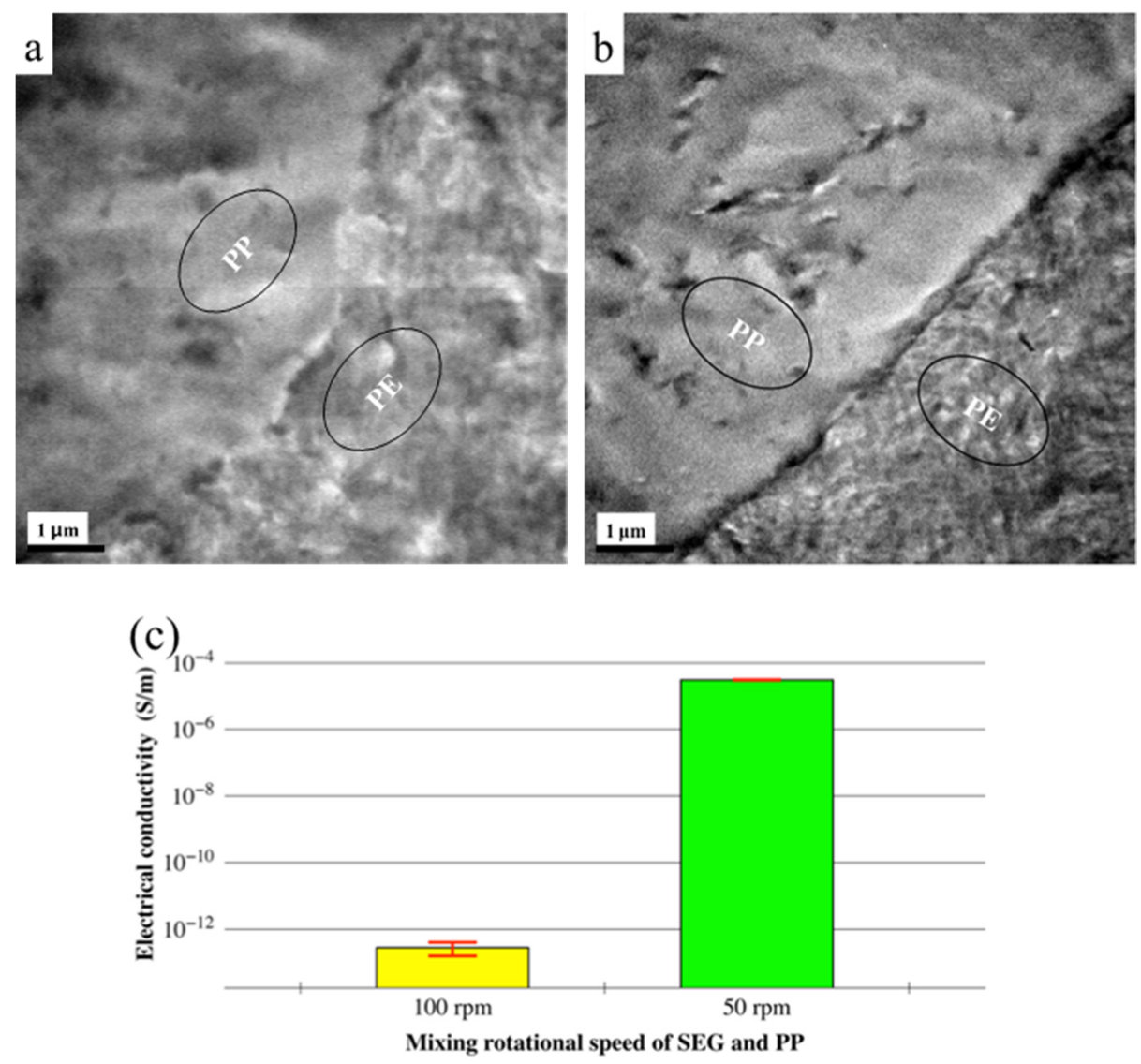

Figure 4. TEM micrographs of $3 \mathrm{wt} \%$ SEG filled (SEG/PP)/PE composites at different mixing rotational speed of SEG and PP in (a) $100 \mathrm{rpm}$, (b) $50 \mathrm{rpm}$ (The PP and PE phases are indicated by the ellipses.), (c) Effect of mixing rotational speed of SEG and PP on electrical conductivities of $3 \mathrm{wt} \%$ SEG filled (SEG/PP)/PE composites

Because of the short processing times, for the (SEG/PP)/PE composites, the SEG transfer situation is highly determined by the melt-mixing time (Kaftelen et al., 2012). Therefore, the simplest way to achieve a optimized localization of SEG at the PE/PP blends interface is increasing mixing times of premixed SEG/PP and PE, in which the SEG be able to fully transferred from PP phase to the interface. Then, the SEG transfer situation can determine the final localization of SEG in the PE/PP blends. Thus, the mixing times of premixed SEG/PP and PE were increased from $10 \mathrm{~min}$ to $15 \mathrm{~min}$ and $20 \mathrm{~min}$. As illustrated in Figure 5a-c, the transfer situation and localization of SEG in the $1 \mathrm{wt} \%$ SEG filled (SEG/PP)/PE composites dependence on mixing time of SEG/PP and PE have been observed by TEM. It could be clear observed that the amounts of dispersed SEG in PP phase was decreased with mixing times of premixed SEG/PP and PE, and a preferable localization of SEG at the PE/PP blends interface was formed (Figure 5c).

Figure $5 \mathrm{~d}$ presents the corresponding electrical conductivity of the composites shown in Figure 5a-c, interpreted the effect of the mixing time of (SEG/PP) and PE on the electrical conductivity. The electrical conductivity of $1 \mathrm{wt} \%$ SEG filled (SEG/PP)/PE composite was $1.86 \times 10^{-5} \mathrm{~S} / \mathrm{m}$ and is indeed observed when the premixed SEG/PP and PE were mixed for $20 \mathrm{~min}$, in sharp contrast to the $4 \mathrm{wt} \%$ SEG needed in originally (SEG/PP)/PE procedure. A low percolation at nearby $1 \mathrm{wt} \%$ SEG was indeed obtained, which is lower than those of many other graphene-filled systems with same loading of graphene by melt compounding (Kim et al., 2011; Yan et al., 2012), and even comparable to those of some systems prepared by solution-mixing (Yoonessi \& Gaier, 2010). When SEG sheets are distributed throughout the PE phase, as opposed to being concentrated at the blends interface, this remarkable reduction in the percolation threshold is never observed upon the increasing mixing times of the blends, and what's more, when the SEG was firstly mixed with less favorable phase PP, the localization of SEG could be optimized located at the interface by processing parameter control. 

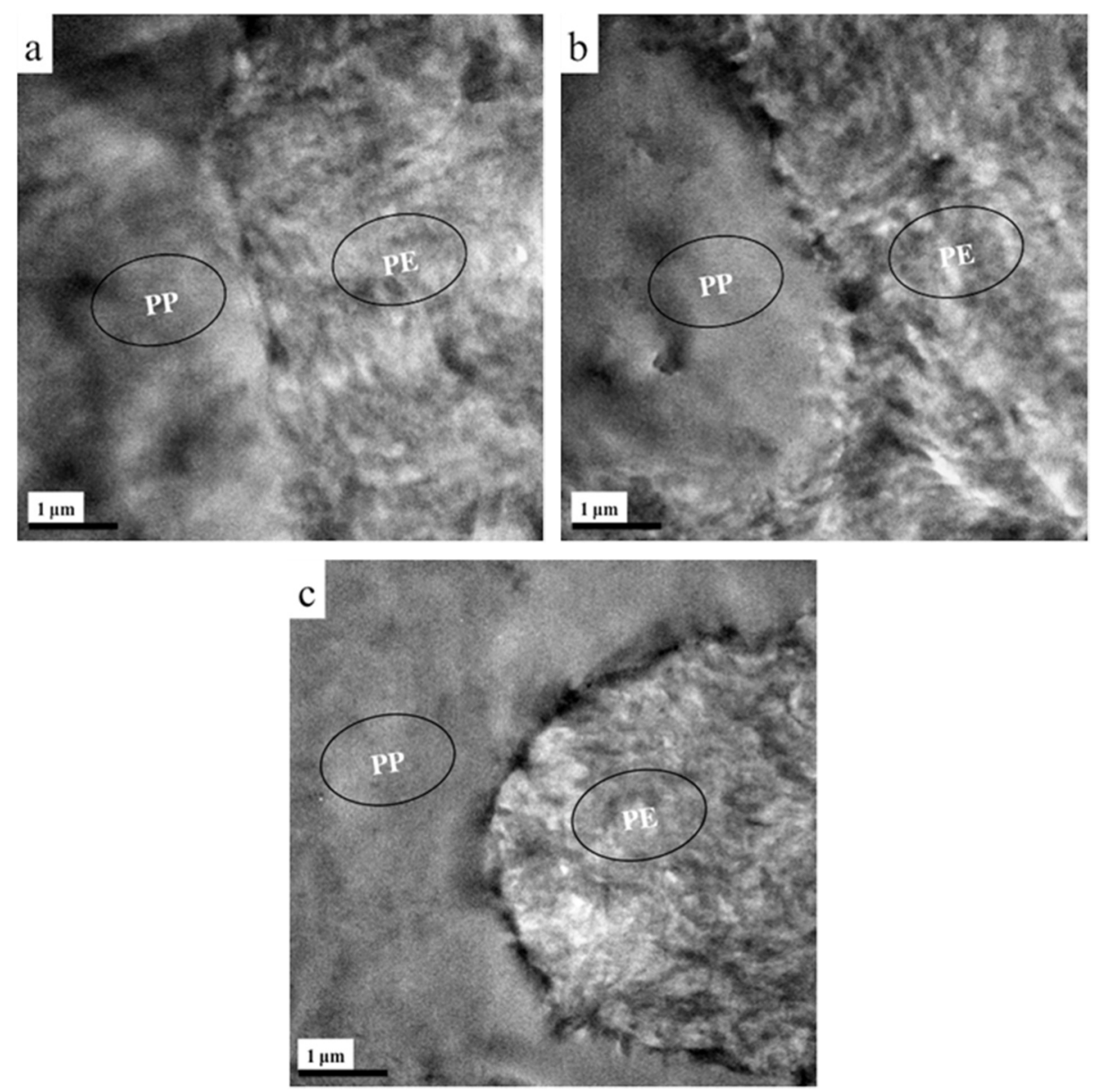

(d)

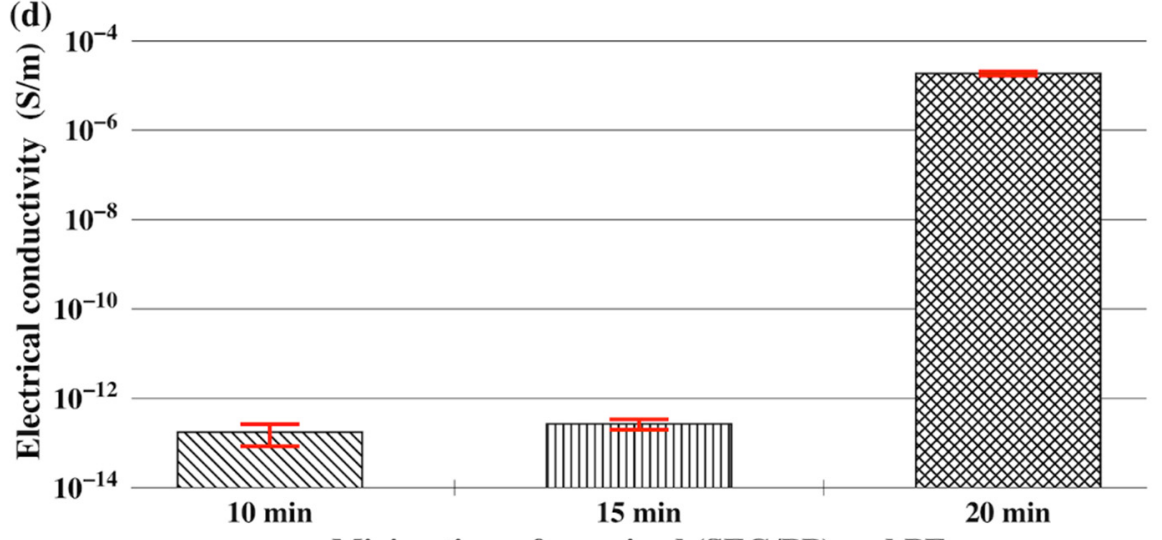

Mixing time of premixed (SEG/PP) and PE

Figure 5. TEM micrographs of the melt mixing time of premixed (SEG/PP) and PE for (a) 10min, (b) 15min, (c) 20 min of PE/PP (60/40) blends filled with $1 \mathrm{wt} \%$ SEG (The PP and PE phases are indicated by the ellipses.), (d) Influence of the melt-mixing time of premixed (SEG/PP) and PE on the electrical conductivity of (SEG/PP)/PE composites filled with $1 \mathrm{wt} \%$ SEG

It was well know that the rheometry is one of the most sensitive methods to probe the microstructure of polymer-nanofiller composites (Solomon, Almusallam, Seefeldt, Somwangthanaroj, \& Varadan, 2001), a variety of storage moduli $\left(G^{\prime}\right)$ at low frequency plateau can be observed when the nanofillers form a particulate network (Zhu., Cardinaels, Mewis, \& Moldenaers, 2009; Krishnamoorti \& Yurekli, 2001). To investigate the effect of graphene localization on the particulate network, the variation of storage modulus $\left(G^{\prime}\right)$ with frequency $(\omega)$ for the (SEG/PP)/PE (1 wt \% SEG) composites at different melt-mixing time of premixed (SEG/PP) and PE is presented in Figure 6. In the low-frequency part, the 20min composite exhibits a higher $G$ ' value than that of the 10 and 15 
min composites. The clear increase of low-frequency $G$ ' at $20 \mathrm{~min}$ composite is mainly contributed by a better localization of graphene at the interface of PE/PP blends (Figure 5c), and thus the graphene was able to build a percolating network with the same loading. As a result, the samples of $20 \mathrm{~min}$ composite shows a much higher rheological properties, which is in good accordance with the electrical properties of the composites.

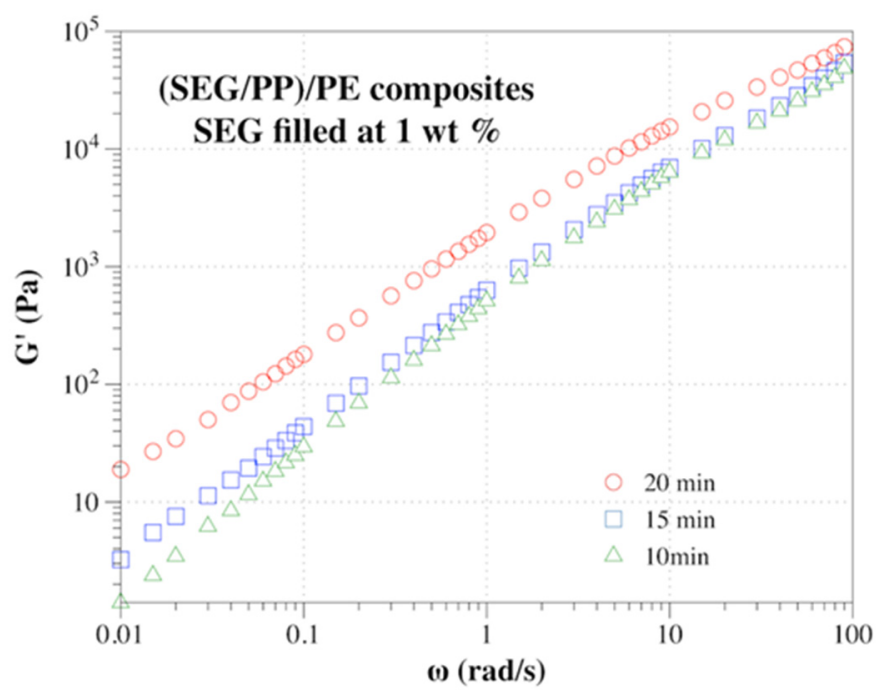

Figure 6. Storage modulus $\left(G^{\prime}\right)$ as a function of frequency $(\omega)$ for $1 \mathrm{wt} \%$ SEG filled (SEG/PP)/PE composites with varied the melt-mixing time of premixed (SEG/PP) and PE

\section{Conclusion}

The experimental results reported in this paper convincingly emphasize that blends of insulating immiscible PE/PP polymer blends can be endowed with electrical conductivity by using very small amounts of conductive SEG fillers. A low percolation at nearby $1 \mathrm{wt} \%$ SEG was observed when the localization of SEG optimized at the $\mathrm{PE} / \mathrm{PP}$ blends interface. Morphology analysis of TEM micrographs has clearly shown the critical importance of properly controlling localization of SEG in the melt-mixing polymer composites. Such procedures indeed prefect the localization of SEG at the PE/PP blends interface. It is responsible for a percolation threshold as low as $1 \mathrm{wt} \%$ SEG, compared to $7 \mathrm{wt} \%$ if the localization of SEG has not been optimized. Our results indicate that the localization of graphene could be design at immiscible polymer blends interface, which is an efficient way to improve the electrical properties. Above all, a comprehensive understanding of graphene localization in polymer composites during melt mixing will be one of the critical factors to create the novel conductive composites.

\section{Acknowledgments}

We acknowledge the use of the Instrument and Research Technology Center at Nagoya Institute of Technology for TEM and AFM analysis.

\section{References}

Abolins, V., Caraher, J., Bopp, R. C., \& Lovgren, E. M. (1983). Eur. Patent, EP 77059. Washington, DC: U.S. Patent and Trademark Office.

Alig, I., Potschke, P., Lellinger, D., Skipa, T., Pegel, S., \& Kasaliwal, G. R. (2012). Establishment, morphology and properties of carbon nanotube networks in polymer melts. Polymer, 53, 4-28. https://doi.org/10. 1016/j.polymer.2011.10.063

Geim, A. K. (2009). Graphene: status and prospects. Science, 324, 1530-1534. https://doi.org/10.1126/science. 1158877

Goldel, A., Marmur, A., Kasaliwal, G. R., Potschke, P., \& Heinrich, G. (2011). Shape-dependent localization of carbon nanotubes and carbon black in an immiscible polymer blend during melt mixing. Macromolecule, 44, 6094-6102. https://doi.org/10.1021/ma200793a 
Gubbels, F., Jerome, R., Vanlathem, E., Deltour, R., Blacher, S., \& Brouers, F. (1998). Kinetic and thermodynamic control of the selective localization of carbon black at the interface of immiscible polymer blends. Chem Mater, 10, 1227-1235. https://doi.org/10.1021/cm970594d

Kaftelen, H., Ocakoglu, K., Thomann, R., Tu, S. Y., Weber, S., \& Erdem, E. (2012). EPR and photoluminescence spectroscopy studies on the defect structure of $\mathrm{ZnO}$ nanocrystals. Phys Rev B, 86, 014113. https://doi.org/ 10.1103/physrevb.86.014113

Kim, H., Miura, Y., \& Macosko, C. W. (2010). Graphene/polyurethane nanocomposites for improved gas barrier and electrical conductivity. Chem Mater, 22, 3441-3450. https://doi.org/10.1021/cm100477v

Kim, H., Abdala, A. A., \& Macosko, C. W. (2010). Graphene/polymer nanocomposites. Macromolecules, 43, 6515-6530. https://doi.org/10.1021/ma100572e

Kim, H., Kobayashi, S., AbdurRahim, M. A., Zhang, M. J., Khusainova, A., Hillmyer, M. A., \& Abdala, A. A. (2011). Graphene/polyethylene nanocomposites: effect of polyethylene functionalization and blending methods. Polymer, 52, 1837-1846. https://doi.org/10.1016/j.polymer.2011.02.017

Krishnamoorti, R., \& Yurekli, K. (2001). Rheology of polymer layered silicate nanocomposites. Curr Opin Colloid Interface Sci, l6, 464-470. https://doi.org/10.1016/s1359-0294(01)00121-2

Nagata, K., Kimura, Y., Takahashi, K., \& Kinoshita, T. (2002). Electrical resistivities of PP/PE composites filled with carbon black/fiber. Jpn J Polym Sci Technol, 59, 694-701. http://doi.org/10.1295/koron.59.694

Novoselov, K. S., Falko, V. I., Colombo, L., Gellert, P. R., Schwab, M. G., \& Kim, K. (2012). A roadmap for graphene. Nature, 490, 192-200. https://doi.org/10.1038/nature11458

Pham, C. V., Repp, S., Thomann, R., Krueger, M., Weberc, S., \& Erdem, E. (2016). Charge transfer and surface defect healing within $\mathrm{ZnO}$ nanoparticle decorated graphene hybrid materials. Nanoscale, 8, 9682-9687. https://doi.org/10.1039/c6nr00393a

Ponomarenko, A. T., Shevchenko, V. G., \& Enikolopyan, N. S. (1990). Formation processes and properties of conducting polymer composites. Adv Polym Sci, 96, 125-147. https://doi.org/10.1007/3-540-52791-5_4

Potschke, P., Bhattacharyya, A. R., \& Janke, A. (2003). Morphology and electrical resistivity of melt mixed blends of polyethylene and carbon nanotube filled polycarbonate. Polymer, 44, 8061-8069. https://doi.org/10.1016/j. polymer.2003.10.003

Rao, C. N. R., Sood, A. K., Subrahmanyam, K. S., \& Govindaraj, A. (2009). Graphene: the new two-dimensional nanomaterial. Angew Chem Int Ed, 48, 7752-7777. https://doi.org/10.1002/anie.200901678

Shannon, M. N. (2012). Highly concentrated aqueous suspensions of graphene through ultrasonic exfoliation with continuous surfactant addition. Langmuir, 28, 14110-14113. https://doi.org/10.1021/la302750e

Shen, Y., Zhang, T. T., Yang, J. H., Zhang, N., Huang, T., \& Wang, W. (2015). Selective localization of reduced graphene oxides at the interface of PLA/EVA blend and its resultant electrical resistivity. Polym Compos. https://doi.org/10.1002/pc.23769

Socher, R., Krause, B., Boldt, R., Hermasch, S., Wursche, R., \& Pötschke, P. (2011). Melt mixed nano composites of PA12 with MWNTs: Influence of MWNT and matrix properties on macrodispersion and electrical properties. Compos Sci Technol, 71, 306-314. https://doi.org/10.1016/j.compscitech.2010.11.015

Solomon, M. J., Almusallam, A. S., Seefeldt, K. F., Somwangthanaroj, A., \& Varadan, P. (2001). Rheology of polypropylene/clay hybrid materials. Macromolecules, 34, 1864-1872. https://doi.org/10.1021/ma001122e

Stankovich, Y. S., Dikin, D. A., Piner, R. D., Kohlhaas, K. A., Kleinhammes, A., \& Jia, Y. Y. (2010). Synthesis of graphene-based nanosheets via chemical reduction of exfoliated graphite oxide. Carbon, 48, 1558-1565. https://doi.org/10.1016/j.carbon.2007.02.034

Thongruang, W., Spontak, R. J., \& Balik, C. M. (2002). Bridged double percolation in conductive polymer composites: an electrical conductivity, morphology and mechanical property study. Polymer, 43, 3717-3725. https://doi.org/10.1016/s0032-3861(02)00180-5

Tu, C., Nagata, K., \& Yan, S. K. (2015). Morphology and electrical conductivity of polyethylene/polypropylene blend filled with thermally reduced graphene oxide and surfactant exfoliated graphene. Polym Compos. https://doi.org/10.1002/pc.23782 
Yan, D., Zhang, H. B., Jia, Y., Hu, J., Qi, X. Y., \& Zhang, Z. (2012). Improved electrical conductivity of polyamide 12/graphene nanocomposites with maleated polyethylene-octene rubber prepared by melt compounding. ACS Appl Mater Interfaces, 4, 4740-4745. https://doi.org/10.1021/am301119b

Yavari, F., Rafiee, M. A., Rafiee, J., Yu, Z. Z., \& Koratkar, N. (2010). Dramatic increase in fatigue life in hierarchical graphene composites. ACS Appl Mater Interfaces, 2, 2738-2743. https://doi.org/10.1021/am $100728 \mathrm{r}$

Yoonessi, M., \& Gaier, J. R. (2010). Highly conductive multifunctional graphene polycarbonate nanocomposites. ACS Nano, 4, 7211-7220. https://doi.org/10.1021/nn1019626

Young, T. (1805). An essay on the cohesion of fluids. Philos Trans R Soc London, 95, 65-87.

Zhu, Y. T., Cardinaels, R., Mewis, J., \& Moldenaers, P. (2009). Rheological properties of PDMS/clay nanocomposites and their sensitivity to microstructure. Rheol Acta, 48, 1049-1058. https://doi.org/10.1007/ s00397-009-0387-3

\section{Copyrights}

Copyright for this article is retained by the author(s), with first publication rights granted to the journal.

This is an open-access article distributed under the terms and conditions of the Creative Commons Attribution license (http://creativecommons.org/licenses/by/4.0/). 